\title{
Development and Analysis of Artificial Neural Network Models for Rainfall Prediction by Using Time-Series Data
}

\author{
Neelam Mishra \\ Department of Computer Science and Engineering, NRI College of Engineering and Management, \\ Gwalior, Madhya Pradesh, India \\ Hemant Kumar Soni \\ Department of Computer Science and Engineering, Amity School of Engineering and Technology, \\ Amity University, Gwalior, Madhya Pradesh, India \\ E-mail: hksoni@gwa.amity.edu \\ Sanjiv Sharma \\ Department of Computer Science and Engineering, Madhav Institute of Technology and Science, \\ Gwalior, Madhya Pradesh, India \\ A K Upadhyay \\ Department of Computer Science and Engineering, Amity School of Engineering and Technology, \\ Amity University, Gwalior, Madhya Pradesh, India
}

Received: 19 May 2017; Accepted: 21 July 2017; Published: 08 January 2018

\begin{abstract}
Time Series data is large in volume, highly dimensional and continuous updating. Time series data analysis for forecasting, is one of the most important aspects of the practical usage. Accurate rainfall forecasting with the help of time series data analysis will help in evaluating drought and flooding situations in advance. In this paper, Artificial Neural Network (ANN) technique has been used to develop one-month and twomonth ahead forecasting models for rainfall prediction using monthly rainfall data of Northern India. In these model, Feed Forward Neural Network (FFNN) using Back Propagation Algorithm and Levenberg- Marquardt training function has been used. The performance of both the models has been assessed based on Regression Analysis, Mean Square Error (MSE) and Magnitude of Relative Error (MRE). Proposed ANN model showed optimistic results for both the models for forecasting and found one month ahead forecasting model perform better than two months ahead forecasting model. This paper also gives some future directions for rainfall prediction and time series data analysis research.
\end{abstract}

Index Terms - Data Mining, Time series data analysis, Rainfall forecasting, Artificial Neural Network, Feed Forward Neural Network.

\section{INTRODUCTION}

Rainfall is recognizing as one of the main important element of the hydrological process. Accurate rainfall forecasting will help in evaluating drought and flooding situations in advance. Therefore, it is significant to have a perfect model for rainfall forecasting.

The country like India, where most of the farmers depend on monsoon for their crop, it is required to be having advance knowledge of actual rainfall. Many states of India also suffered from the flood whereas some state is facing the problem of drought. In any of these two situations, it must require an accurate and efficient rainfall prediction model. This kind of rainfall forecasting model will help in better handling worst situations generated due to flood or draught. Advance prediction of rainfall by this model also gives sufficient time to makes adequate arrangements for saving lives, transportation, procurement and supply of food and medicines.

Data mining is a set of techniques used to extract unknown pieces of information from the large database repository. There are various data mining techniques available to extract valuable and useful information from spatial, temporal, sequencing and time series data.

Time Series data is a part of temporal data. Time series data generated from scientific data, financial applications, weather data, GPS, Sensor Networks etc. Large in volume, highly dimensional and continuous updating is the nature of time series data [1]. Use of time series data [2] in prediction, pattern identification, anomaly detection, motif discovery, clustering, classification, segmentation fetches the attention of data mining researchers.

In time series data analysis the patterns which existed in past will appear in future also. This time series data analysis allows a model for a complex system where the 
goal is to predict the system's behavior without knowing the functionality of the system [3].

There are various statistical techniques, data mining approaches, artificial neural network and soft computing techniques including particle swarm optimization used by the researcher to solve time series data analysis problem $[4,5]$.

Artificial Neural Networks (ANN) is an extensively established technique for modeling complex nonlinear and dynamic systems. ANN is helpful in developing an appropriate model when physical process relationship is not clear or where the nature of the event has chaotic properties. Although neural network required prior information about the system but it reduces the model's reliance on this prior information. This removes the need for an exact specification of the precise functional form of the relationship that the model seeks to represent. In this paper, an attempt has been made to develop a 1month and 2-month rainfall forecasting model for rainfall prediction in North India by using ANN.

This paper is divided into 7 sections. First Section cover introduction, section two discuss the related work in this domain. The third section describes objective and significance of the study. Section forth explain the data set used. The fifth section describes ANN model development and section 6 cover result and discussion. Paper end with a conclusion, limitations and future scope by section 7 .

\section{RELATED WORK}

There are various methodologies used for time series data analysis in which implementation of Neural Network $(\mathrm{NN})$ is a popular choice for the researchers.

Various methodologies have been used for time series data analysis by researchers in which performance of Neural Network (NN) is accepted and best option for the researchers amongst the different techniques like Generalized Regression, Pearson Coefficient Technique, Fuzzy Inference System, Focused Time Daley Neural Network etc. Various work has been carried out by researchers on time series data analysis. In this regard, the model was developed by using Generalized Regression Neural Network [6] and compared with the model based on the Back propagation Neural Network. The Generalized Regression Neural Network yielded simple and stable structure, with an accurate prediction of annual rainfall. Pearson Coefficient Technique [7] was compared with Regression Approach and found that the predicted values are lie below computed value for time series data analysis. Apart from above techniques, Fuzzy Inference System [8] was observed as a good alternative method for accurate prediction. Accurate results can also be obtained by using Focused Time Daley Neural Network model [9]. The model's predictions were on monthly, quarterly and annually basis. NNARX and ANFIS [10] models were also found accurate for rainfall-runoff prediction and reliable forecasting. Model using Neuro-Fuzzy Neuron Technique [11] was designed and compared with Dynamic Downscaling Model. It is found that model produced improved results with low computation cost for seasonal rainfall forecast. In continuation with previous models, The ANN and Wavelets Decomposition model [12] for rainfall predictions, CART and C4.5 [13] for hourly rainfall predictions were also developed by researchers for forecasting. Amongst all improved Naive Bayes Classifier for time series data analysis had accuracy rate $90 \%$ on rain /no-rain classification in the comparison of genetic algorithm with average classification technique[14,15].

Awan et al. [16] proposed Back Propagation (BP) and Learning Vector Quantization (LVQ) technique for rainfall prediction. Author claim their techniques perform better in terms of accuracy, lead time and required fewer resources. The also assert that LVQ takes less training time than Back Propagation. Du et al. [17] suggested Immune Evolutionary Algorithm based on Back propagation network. Authors said their model have higher accuracy and better stability. The author also claims their model is suitable for solving complicated problems of optimization. Jin et al. [18] proposed Particle Swarm Optimization (PSO) - Neural Network ensemble prediction model for monthly mean rainfall. Authors compared their result with traditional linear statistical forecast method and claim superior predicting capability and enhanced generalization capacity. Suhartono et al. [19] implemented ensemble method based on ANFIS and ARIMA for monthly rainfall prediction. Decision Tree Method using SLIQ [20] generated classification rule for rainfall prediction with $72.3 \%$ accuracy. Feed-forward NN based NAR model [21] for forecasting time series was also an important outcome for forecasting.

Genetic Algorith [22] and Artificial Neural Network [23, 24,25,26], Multi-Layer Perceptron (MLP), Functional Link Artificial Neural Network (FLANN) and Legendre Polynomial Equation (LPE) [27], Multiple Linear Regression (MLR) techniques [28] were introduced for Rainfall prediction.

\section{OBJECTIVE AND SIGNIFICANCE OF THE STUDY}

The prime objective of this study is to analyze rainfall pattern in North India using appropriate time series methods for the period 1871 to 2012 ( 141 years) on the basis of data recorded at Indian Meteorological Department, Pune. The specific objectives are:

1. To develop a time series model for one-month and two-month ahead rainfall forecasting

2. To identify the best network structure for both the models.

3. Compare the performance of one-month and twomonth ahead forecasting models.

Weather and climate over the earth are not constant with time, they change on different time series ranging from the geological to the diurnal through annual, seasonal and intra-seasonal timescales. Rainfall varies with latitude, elevation, topography, seasons, distance from the sea, and Coastal sea-surface temperature. Trends 
in rainfall extremes have enormous implications. Flood or draught events cause significant damage to agriculture, environment, infrastructure, social life and on GDP. Therefore, a reliable rainfall forecasting and assessing behavior at the station, regional and national levels is very important. The results of this research paper will hopefully be used:

1. For forecasting the pattern of rainfall in the study area.

2. To provide information that would be helpful for decision makers in formulating policies to mitigate the problems of rainwater resources management, soil erosion, flooding, and drought.

3. To provide information for the early warning system in the study area.

4. As a basis for further study in Northern India.

\section{ANN MODEL DEVELOPMENT}

Artificial Neural Network (ANN) Model is developed using MATLAB. In this model Feed Forward Neural Network (FFNN) using Back Propagation algorithm and Levenberg- Marquardt training function has been used. Trials are first conducted by randomly selecting the number of processing elements at the end of the training, on the basis of a mean square error, accuracy will be predicted. As per the performance of model and resultant predictions, the selection of the processing elements carefully narrowed down. This ANN model generates a very accurate prediction for the range of 5 to 40 neurons. This ANN model consists of one hidden layer and a varying number of processing elements or neurons. The most optimal model obtained by using trial and error approach. The training parameters applicable for model training are given in the Table-1.

Table 1. Model parameter values for Back Propagation Algorithm for all the models

\begin{tabular}{|l|l|}
\hline \multicolumn{2}{|l|}{ Parameters used for Network Training } \\
\hline Network Type & Feed Forward Neural Network with Back Propagation \\
\hline Training Functions Used & Levenberg-Marquardt (LM) \\
\hline Adoption Learning Function & learnGDM and learnGD \\
\hline Performance Function & Mean Square Error (MSE); Regression (R) \\
\hline Transfer Function & $\begin{array}{l}\text { For Hidden Layer - Tansigmoid } \\
\text { For Output Layer - Linear }\end{array}$ \\
\hline No. of neurons used for hidden layer & 5 to 40 \\
\hline
\end{tabular}

\section{DATA SET UseD}

For this one-month and two-month ahead rainfall forecasting model development, a monthly time series rainfall data of North India for the period 1871 to 2012 ( 141 years) were used. This time series data collected by Indian Meteorological Department, Pune. The data were collected by various stations of different states of Northern India. The site was chosen due to the availability of relatively long series of meteorological data.

After analyzed data, some data was selected to train ANN models, and the remaining was used as a testing set.
This study focuses on the North India only, so only stations located in this area were selected, while the other stations which are located outside were discarded. The data used as input and output variables for optimum model development are given in Table 2 below. Here two models shall be developed, model M1 is for onemonth ahead prediction and the other model M2, is for two-month ahead prediction. In both M1 and M2 models, three input variables have been used which include successive months of rainfall data. The output in both the models is the predicted rainfall, which is one month and two month ahead values.

Table 2. Model Input / Output Variables

\begin{tabular}{|c|c|c|}
\hline Model & Input Variables Rainfall (mm) & Output Variables Rainfall (mm) \\
\hline \multirow{3}{*}{ M1 } & $\mathrm{R}(\mathrm{t}-2)$ & \multirow{2}{*}{$\mathrm{R}(\mathrm{t}+1)$} \\
\cline { 2 - 2 } & $\mathrm{R}(\mathrm{t}-1)$ & \\
\cline { 2 - 2 } & $\mathrm{R}(\mathrm{t})$ & \multirow{2}{*}{$\mathrm{R}(\mathrm{t}+2)$} \\
\cline { 2 - 2 } M2 & $\mathrm{R}(\mathrm{t}-2)$ & \\
\cline { 2 - 2 } & $\mathrm{R}(\mathrm{t}-1)$ & \\
\hline
\end{tabular}

\section{RESULTS AND DISCUSSIONS}

Network performance of both the models M1 and M2 are given in tables below. In Table 3 and Table 4, the number of neurons in the hidden layer is arranged in ascending order and their validation performance is measured accordingly. 
Table 3. Network Performance for Model M1 for one Month Ahead Forecasting

\begin{tabular}{|c|c|c|c|c|}
\hline Neurons Used & Model Performance & $\begin{array}{c}\text { No. of Iteration } \\
\text { (Epochs) }\end{array}$ & Stopping Criteria & Network Configuration \\
\hline 5 & 120914.77 & 12 & Val. Stopped/Max. iterations & $3-5-1$ \\
\hline 10 & 120332.90 & 14 & Val. Stopped/Max. iterations & $3-10-1$ \\
\hline 15 & 129833.71 & 22 & Val. Stopped/Max. iterations & $3-15-1$ \\
\hline 20 & 136442.98 & 8 & Val. Stopped/Max. iterations & $3-20-1$ \\
\hline $\mathbf{2 5}$ (best) & $\mathbf{1 1 2 4 6 1 . 9 5}$ & $\mathbf{4}$ & Val. Stopped/Max. iterations & $3-30-1$ \\
\hline 30 & 152499.50 & 23 & Val. Stopped/Max. iterations & $3-35-1$ \\
\hline 35 & 103683.66 & 24 & Val. Stopped/Max. iterations & $3-40-1$ \\
\hline 40 & 122817.65 & 13 & Val. Stopped/Max. iterations & $\mathbf{3 - 4 5 - 1}$ \\
\hline $\mathbf{4 5}$ (best) & $\mathbf{1 3 2 3 7 3 . 4 2}$ & $\mathbf{8}$ & Val. Stopped/Max. iterations & V-50-1 \\
\hline 50 & 154452.31 & 6 & Val. Stopped/Max. iterations & \\
\hline
\end{tabular}

Table 4. Network Performance for Model M2 for two Month Ahead Forecasting

\begin{tabular}{|c|c|c|c|c|}
\hline Neurons Used & Model Performance & $\begin{array}{c}\text { No. of Iteration } \\
\text { (Epochs) }\end{array}$ & Stopping Criteria & Network Configuration \\
\hline 5 & 196653.42 & 34 & Val. Stopped/Max. iterations & $3-5-1$ \\
\hline 10 & 169111.91 & 44 & Val. Stopped/Max. iterations & $3-10.1$ \\
\hline 15 & 142875.66 & 29 & Val. Stopped/Max. iterations & $3-15-1$ \\
\hline 20 & 146643.47 & 12 & Val. Stopped/Max. iterations & $3-20-1$ \\
\hline 25 & 198612.44 & 11 & Val. Stopped/Max. iterations & $3-25-1$ \\
\hline 30 & 220510.21 & 8 & Val. Stopped/Max. iterations & $3-30-1$ \\
\hline 35 & 208755.18 & 5 & Val. Stopped/Max. iterations & $3-35-1$ \\
\hline 40 & 233804.28 & 10 & Val. Stopped/Max. iterations & $3-40-1$ \\
\hline 45 & 159821.84 & 13 & Val. Stopped/Max. iterations & $3-45-1$ \\
\hline $\mathbf{5 0}$ (best) & $\mathbf{1 3 8 6 4 3 . 4 7}$ & $\mathbf{1 2}$ & Val. Stopped/Max. iterations & $\mathbf{3 - 5 0 - 1}$ \\
\hline
\end{tabular}

It is observed that for Model M1 and M2, the best network structure is 3-25-1 and 3-50-1 respectively. It is also found out that the performance is not necessarily improved even when the network error is low.

It is very much clear from figure $1(\mathrm{a})$ and $1(\mathrm{~b})$ that

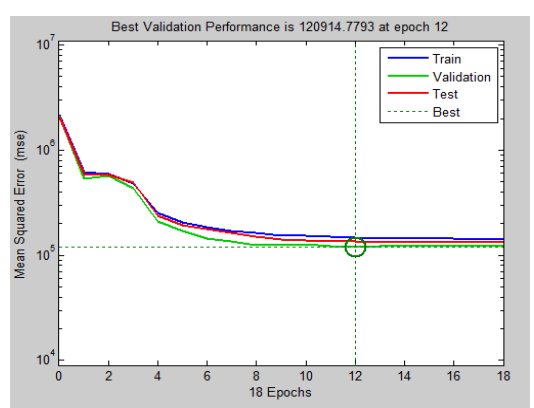

(a) after 12 and 9 epochs, the performance of training, testing and validation errors were stagnant. It means after epochs 12 and 9 there is no further improvement in the performance of the network and the network seems to have saturated.

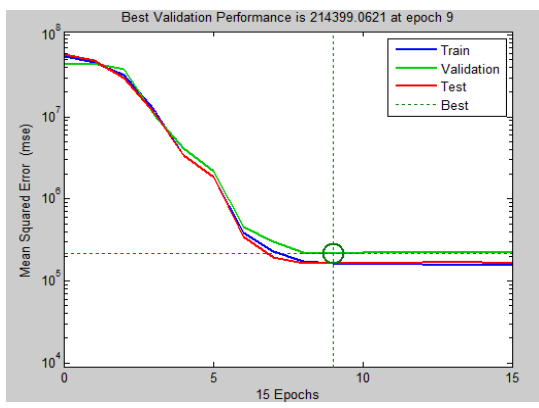

(b)

Fig.1. Training of NN Model gauged by MS for M1 and M2 Models respectively.

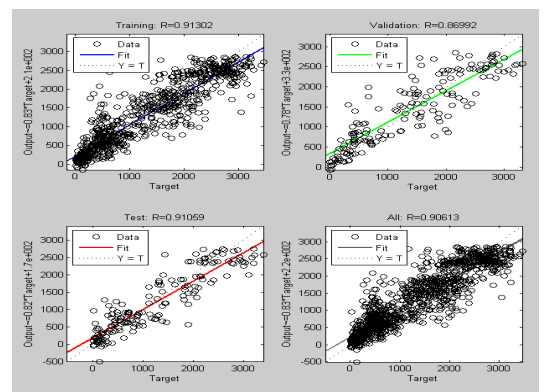

(a) for $\mathrm{N}=45$

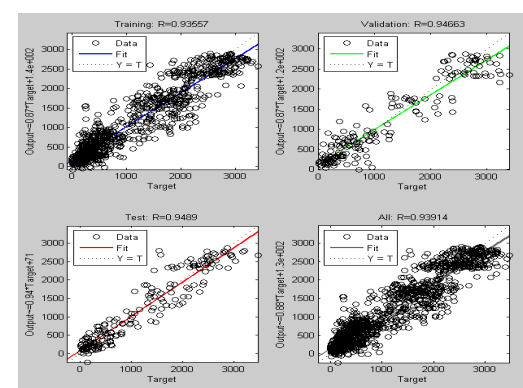

(b) for $\mathrm{N}=50$

Fig.2. Scatter Plot of R-Values for Training, Testing and Validating datasets for best-developed network for M1 and M2 Models Respectively 
Linear regression analysis between the network response and the network output shown in figure 2. It can be inferred that $\mathrm{NN}$ model does the good mapping. In this study, $15 \%$ data used for validation purpose, which is other than training data for the model. Thus, the performance of these machining conditions never experienced before by the neural network model. Hence, this mapping considers to be true and representing the functional relationship.

Following figures 3 given below depicts the comparison between actual and simulated data for surface roughness. It is noticed that except for rare occasions, simulated surface roughness values for the designated parameters are in acceptable proximity with actual values. This representation, therefore, agrees with the conclusion that, high accuracy of prediction is attained by Neural Network Model after successful completion of training criteria i.e. with the value of MSE being within an acceptable range as well as an agreeable performance measure. Hence, from the results, it is inferred that the performance of the NN model is acceptable. Thus, can further be confirmed from the scatter plots shown in 4 . Further analysis of the observed and predicted values for both M1 and M2 models on the basis of MRE values are shown in figures 5 .

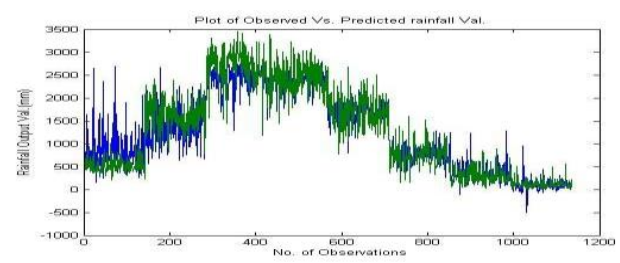

(b)

Fig.3. Comparison of observed and predicted values by best M1 \& M2 models

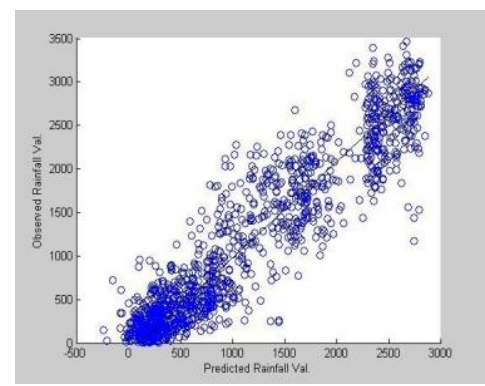

(a)

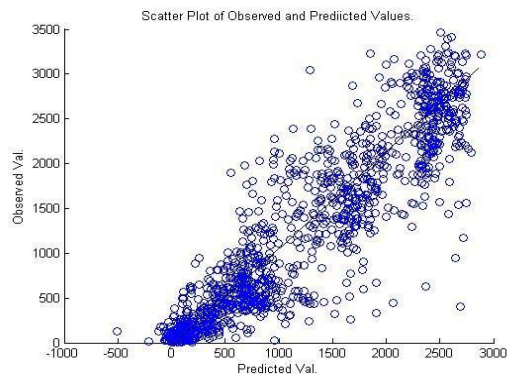

(b)

Fig.4. Scatter Plot of Observed vs. Predicted Rainfall values for M1 \& M2 Models

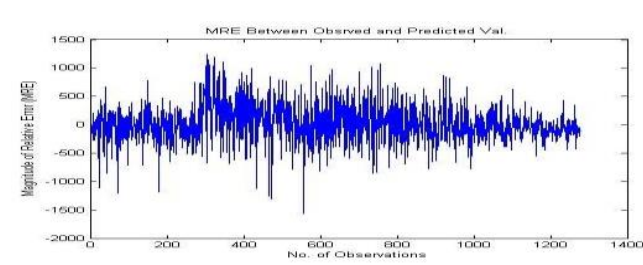

(a)

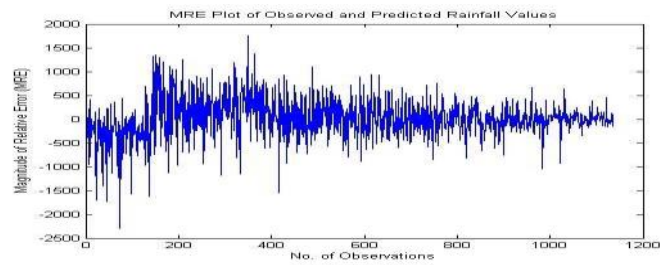

(b)

Fig.5. Deviation of Predicted value from Observed value for M1\& M2 Models

Computed Regression and MSE values for Model M1 and Model M2 for different network structures are given in Table 3 and Table 4 respectively. For the network identification purpose, as given in the last column, the first number indicates the number of neurons in the input layer, middle represents neurons in the hidden layer and the last number indicates the number of neurons in the output layer.

\section{A. The performance of M1 Model:}

As shown in Table 3, M1 Model gives the best result in 3-25-1structure, with the least MSE value of 112461.95 and the best regression values of 0.946 and 0.948 for the validating and testing data sets respectively. Although the regression values are slightly inferior for training data in the comparison of validation data regression value and testing data regression value. The same has been depicted graphically in figure 6 given below. 
Table 5. Statistical Parameters for different network structures for M1 Model

\begin{tabular}{|c|c|c|c|c|c|}
\hline \multirow{2}{*}{ Structure No. } & \multirow{2}{*}{ No. of Neurons } & \multicolumn{3}{|c|}{ Regression Values } & MSE \\
\hline & & Training Val. & Validating Val. & Testing Val. & All \\
\hline 1 & $3-5-1$ & 0.9211 & 0.927 & 0.93 & 0.923 \\
\hline 2 & $3-10-1$ & 0.936 & 0.937 & 0.941 & 0.937 \\
\hline 3 & $3-15-1$ & 0.943 & 0.931 & 0.92 & 0.937 \\
\hline 4 & $3-20-1$ & 0.946 & 0.921 & 0.916 & 0.937 \\
\hline $\mathbf{5}$ & $\mathbf{3 - 2 5 - 1}$ & $\mathbf{0 . 9 3 5}$ & $\mathbf{0 . 9 4 6}$ & $\mathbf{0 . 9 4 8}$ & $\mathbf{0 . 9 3 9}$ \\
\hline 6 & $3-30-1$ & 0.948 & 0.912 & 0.929 & 0.939 \\
\hline 7 & $3-35-1$ & 0.95 & 0.941 & 0.922 & 0.945 \\
\hline 8 & $3-40-1$ & 0.951 & 0.931 & 0.93 & 0.945 \\
\hline 9 & $3-45-1$ & 0.942 & 0.936 & 0.943 & 0.942 \\
\hline 10 & $3-50-1$ & 0.949 & 0.916 & 0.937 & 0.941 \\
\hline
\end{tabular}

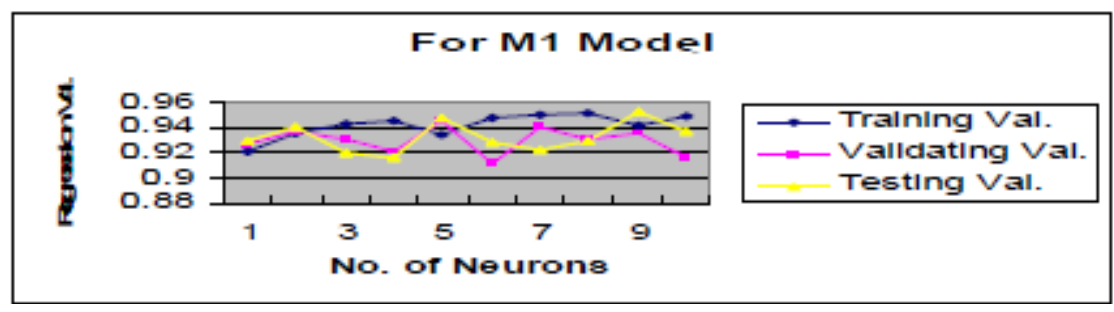

Fig.6. Graphical representation of Regression values for M1 Model

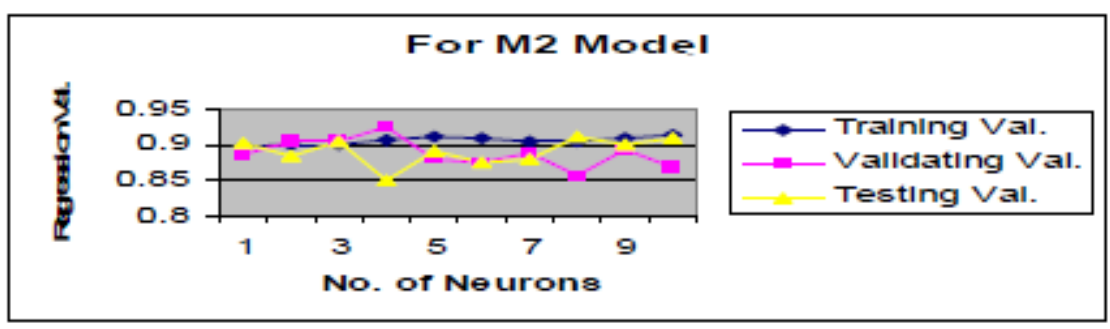

Fig.7. Graphical representation of Regression values for M2 Model

Further from table 3 and figure 3(b), it is observed that, in general, the $\mathrm{R}$ values for training are in general better than testing and validating datasets, which shows that the network seems to have performed well for training datasets. Further, from the detailed analysis of figure 3, it is observed that as the size of the network structure increases, the R-value for training dataset becomes better but there is a slight fall in the values of $\mathrm{R}$ for testing and validating datasets.

Further, a comparative analysis of M1 and M2 model based on tables 5 and 6 shows that M1 model for onemonth ahead rainfall, values have better $\mathrm{R}$ for training, testing and validating datasets than an M2 model for two- months ahead rainfall prediction.

Table 6. Statistical Parameters for different network structures for M2 Model

\begin{tabular}{|c|c|c|c|c|c|}
\hline \multirow{3}{*}{$\begin{array}{c}\text { Structure } \\
\text { No. }\end{array}$} & \multirow{3}{*}{$\begin{array}{c}\text { No. of } \\
\text { Neurons } \\
\end{array}$} & \multirow{2}{*}{\multicolumn{3}{|c|}{ Regression Values }} & \multirow{3}{*}{$\begin{array}{c}\text { MSE } \\
\text { All }\end{array}$} \\
\hline & & & & & \\
\hline & & $\begin{array}{c}\text { Training } \\
\text { Value }\end{array}$ & $\begin{array}{c}\text { Validating } \\
\text { Value }\end{array}$ & $\begin{array}{l}\text { Testing } \\
\text { Value }\end{array}$ & \\
\hline 1 & $3-5-1$ & 0.897 & 0.886 & 0.903 & 0.896 \\
\hline 2 & $3-10-1$ & 0.901 & 0.904 & 0.884 & 0.900 \\
\hline 3 & 3-15-1 & 0.901 & 0.904 & 0.905 & 0.902 \\
\hline 4 & 3-20-1 & 0.907 & 0.924 & 0.849 & 0.901 \\
\hline 5 & $3-25-1$ & 0.912 & 0.879 & 0.892 & 0.904 \\
\hline 6 & $3-30-1$ & 0.908 & 0.876 & 0.875 & 0.899 \\
\hline 7 & $3-35-1$ & 0.905 & 0.889 & 0.88 & 0.899 \\
\hline 8 & $3-40-1$ & 0.905 & 0.856 & 0.909 & 0.899 \\
\hline 9 & $3-45-1$ & 0.909 & 0.894 & 0.901 & 0.905 \\
\hline 10 & $3-50-1$ & 0.913 & 0.869 & 0.910 & 0.906 \\
\hline
\end{tabular}




\section{B. The performance of M2 Model:}

As shown in Table 4, M2 Model gives the best result in 3-50-1structure, with the least MSE value of 138643.47 and the best regression values of 0.913 and 0.910 for the training and testing datasets respectively, with overall Rvalue of 0.906 . Although the regression values are slightly inferior for validation data in the comparison of training data regression value and testing data regression value. The same has been depicted graphically in figure 7 .

\section{CONCLUSION, LIMITATIONS AND FUTURE WORK}

\section{A. Conclusion}

In this study, 141 years rainfall records were analyzed, using data from Indian Meteorological Department, Pune from various weather stations in the North India. Artificial Neural Network (ANN) technique has been used to develop one-month and two- month ahead forecasting models for rainfall prediction using monthly rainfall data. In these models, Feed Forward Neural Network (FFNN) using Back Propagation algorithm and Levenberg- Marquardt training function has been used. The performance of both the models have been assessed based on Regression Analysis, Mean Square Error (MSE) and Magnitude of Relative Error (MRE).Based on the overall results of the research, the following conclusions could be drawn:

1. Two models M1 and M2, for one month and two months ahead prediction of rainfall, having different input variables were trained and tested using ANN technique.

2. In both the cases for M1 and M2 models, 3-25-1 and 3-50-1 network structure model was found to be the best forecasting model.

3. ANN has performed better for M1 model than M2 model, showing that ANN technique has been able to perform better for one month than two months ahead.

4. The regression values for the M1 model have shown better results than the M2 model.

\section{B. Limitations of the Study}

1. This study is based on the data of Northern part of India, which does not cover the entire nation.

2. Rainfall varies with latitude, elevation, topography, seasons, distance from the sea, and coastal Seasurface temperature. In these two models, these constraints are not considered.

3. These models are for only one-month and twomonth ahead prediction. It does not cover the entire monsoon period or for a complete year.

\section{Future Work}

1. An Alert and Decision Support System can be developed which can be helpful in flood or draught situation.

2. A future study can be incorporated multiple parameters for better predictions.

3. In a further study, the models may be developed for the entire nation by using monthly, monsoon and annual data of entire country.

4. The effect, impact and relation to Global Warming and climate change on rainfall may be a hot issue for future research.

5. To improve the efficiency of proposed system, an online learning method for a neural network may be used.

\section{REFERENCES}

[1] Fu Tak-chung. "A review on time series data mining". Engineering Applications of Artificial Intelligence. vol. 24, pp 164-181, 2011.

[2] P. Esling, C. Agon. "Time-Series data mining". ACM Comput. Surv. vol. 45, 1, (12),pp. 1-34, 2012.

[3] "Time series analysis", Ramasubramanian V, IASRI Report. IASRI, Library Avenue, New Delhi, 2007

[4] Neelam Mishra, Hemant Kumar Soni, Sanjiv Sharma. "A Comprehensive Survey of Data Mining Techniques on Time Series Data for Rainfall Prediction”. Journal of ICT Research and Application, in press. 2017

[5] N. Filzah, M. Radzuan, Z. Othman, A. Abu Bakar. "Uncertain Time Series in Weather Prediction". Procedia Technology, vol 11, pp. 557 - 564, 2012.

[6] W. Zhi-liang, S. Hui-hua. "Rainfall Prediction Using Generalized Regression Neural Network: Case study Zhengzhou". International Conference on computational and Information Sciences. pp.1265-1268, 2010.

[7] M Kannan, S Prabhakaran, P. Ramachandran. "Rainfall Forecasting Using Data Mining Technique". International Journal of Engineering and Technology. Vol. 2(6), pp. 397-401, 2010.

[8] K. Jesada, W. K. Wai, F. C. Che. "Rainfall Prediction in the Northeast Region of Thailand Using Modular Fuzzy Inference System". World Congress on Computational Intelligence., vol 10(15), pp. 136-141, 2012.

[9] R Shamin M A, D Han, J Mathew. "ANFIS and NNARX based Rainfall-Runoff Modeling". IEEE International Conference on Systems Man and Cybernetics, pp. 14541459, 2008.

[10] K. K. Htike, O. O. Khalifa. "Rainfall Forecasting Models Using Focused Time-delay Neural Networks". International Conference on Computer and Communication Engineering. vol. 11(13), pp. 1-6, 2010.

[11] T. N. Castro, S. Francisco, JMB Alves, RST Pontes, MBM Firmino, TM Pereira. "Seasonal Rainfall Forest using a Neo-Fuzzy Neuron Model. IEEE International Conference on Industrial Informatics (INDIN), pp. 694698, 2011.

[12] W Phusakulkajorn, C Lursinsap, J Asavanant. "WaveletTransform Based Artificial Neural Network for Daily Rainfall Prediction in Southern Thailand". 9th International Symposium on Communications and Information Technology, Icheon, pp. 432-437, 2009.

[13] J. Soo-Yeon, S. Sharma, Y. Byunggu, J. D. Hyun. "Designing a Rule-Based Hourly Rainfall Prediction Model”. IEEE IRI, pp. 303-308, 2012.

[14] N.A. Charaniya, S.V. Dudul. "Committee of Artificial Neural Networks for Monthly Rainfall Prediction using Wavelet transform". International Conference on Business, Engineering and Industrial Applications, pp. 125-129, 2012.

[15] JNK Liu, BNL Li, TS Dillon. "An Improved Naïve 
Bayesian Classifier Technique Coupled with a Novel Input Solution Method", IEEE Transactions on systems, man, and Cybernetics - Part C: Applications and Reviews. Vol 31(2), pp.249-256, 2001.

[16] JA Awan, O. Maqbool. "Application of Artificial Neural Networks for Monsoon Rainfall Prediction". Sixth International Conference on Emerging Technologies, pp. 27-32, 2010.

[17] D. Jiaxing, Z. Bin , M. Shaohui. "An application on the Immune Evolutionary Algorithm based on Back Propagation in the Rainfall Prediction". International Conference on Computer Science and Electronics Engineering, pp. 313-317, 2012.

[18] J. Long, H. Ying, Zhao , Hua-sheng. "Ensemble Prediction of Monthly Mean Rainfall with a Particle Swarm Optimization - Neural Network Model". IEEE IRI pp. 287-294, 2012.

[19] S. R. Faulina, D. A. Lusia, B. W.Otok, Sutikno and Heri Kuswanto. "Ensemble Method based on ANFIS-ARIMA for Rainfall Prediction". International Conference on Statistics in Science, Business, and Engineering (ICSSBE), pp. 1-4, 2012.

[20] N.Prasad, P. Kumar , MM Naidu. “An Approach to Prediction of Precipitation Using Gini Index in SLIQ Decision Tree". 4th International Conference on Intelligent Systems, Modeling and Simulation, pp. 56-60, 2013.

[21] R. Adhikari, RK Agrawal. "Forecasting strong seasonal time series with Artificial Neural Network". Journal of Scientific and Industrial Research, vol. 71, pp. 657-666, 2012.

[22] S. Singh,J. Gill, "Temporal Weather Prediction using Back Propagation based Genetic Algorithm Technique", I.J. Intelligent Systems and Applications, vol.6 (12), pp. 55-61,2014.

[23] B.M. Al-Maqaleh, A.A. Al-Mansoub and F.N. Al-Badani, "Forecasting using Artificial Neural Network and Statistics Models", I.J. Education and Management Engineering, vol.3, pp. 20-32, 2016.

[24] V. Nourani, T.R. Khanghah, and A.H. Baghanam, "Application of Entropy Concept for Input Selection of Wavelet-ANN Based Rainfall-Runoff Modeling", Journal of Environmental Informatics, vol. 26 (1), pp.52-70, Sep. 2015.

[25] R.C. Deo, M. Sahin, "Application of the Artificial Neural Network model for prediction of monthly Standardized Precipitation and Evapotranspiration Index using hydrometeorological parameters and climate indices in eastern Australia", Atmospheric Research, vol. 161, pp. 65-81, August 2015.

[26] M. Valipour, "Optimization of neural networks for precipitation analysis in a humid region to detect drought and wet year alarms", Meteorological Applications, vol. 23 (1), pp. 91-100, January 2016.

[27] SK Nanda, DP Tripathy, SK Nayak, S Mohapatra. "Prediction of Rainfall in India Using Artificial Intelligent", Systems and Applications.; vol.12, pp.1-22, 2013.

[28] N. Sethi, K. Garg. "Exploiting Data Mining Technique for Rainfall Prediction". International Journal of Computer Science and Information Technologies. Vol. 5 (3), pp.3982-3984, 2014.

\section{Authors' Profiles}

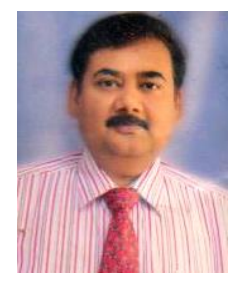

Hemant Kumar Soni received M.Sc. in Computer Science from Jiwaji University, Gwalior, Madhya Pradesh, India in the year 1996 and M. Tech (IT) from Bundelkhand University, Jhansi, Uttar Pradesh, India in the year 2006. He is pursuing Doctoral degree in Computer Science and Engineering from Amity University Madhya Pradesh, Gwalior, India. He has 21 years of teaching experience for UG and PG courses in Computer Science and presently working as Head of the Department of Computer Science and Engineering at Amity University, Gwalior, Madhya Pradesh, India. His research interest in Data Mining and Soft Computing. He published many research papers in National, International Conferences and Scopus Indexed Journals. He is Reviewer of many referred journals. He received a Best Paper Award in an International Conference and organized number of National level events and conferences. He is a member of International Association of Engineers, Hongkong, Universal Association of Computer and Electronics Engineers (UACEE), The Institute of Research Engineers and Doctors, USA, Life Member of ISTE (Indian Society for Technical Education), India and Member of IAENG Society of Computer Science and Data Mining, Hong King.

How to cite this paper: Neelam Mishra, Hemant Kumar Soni, Sanjiv Sharma, A K Upadhyay, "Development and Analysis of Artificial Neural Network Models for Rainfall Prediction by Using Time-Series Data", International Journal of Intelligent Systems and Applications(IJISA), Vol.10, No.1, pp.16-23, 2018. DOI: $10.5815 /$ ijisa.2018.01.03 